\title{
Chaos of Wilson loop from string motion near black hole horizon
}

\author{
Koji Hashimoto and Keiju Murata \\ Department of Physics, Osaka University, Toyonaka, Osaka 560-0043, Japan \\ Norihiro Tanahashi \\ Institute of Mathematics for Industry, University of Kyushu, \\ 744 Motooka, Nishi-ku, Fukuoka 819-0395, Japan
}

(Received 2 April 2018; published 8 October 2018)

\begin{abstract}
To find the origin of chaos near the black hole horizon in string-theoretic AdS/CFT correspondence, we perform a chaos analysis of a suspended string in AdS black hole backgrounds. It has a definite CFT interpretation: chaos of Wilson loops or, in other words, sensitive time evolution of a quark-antiquark force in thermal gauge theories. Our nonlinear numerical simulation of the suspended Nambu-Goto string shows chaos, which would be absent in a pure AdS background. The calculated Lyapunov exponent $\lambda$ satisfies the universal bound $\lambda \leq 2 \pi T_{\mathrm{H}}$ for the Hawking temperature $T_{\mathrm{H}}$. We also analyze a toy model of a rectangular string probing the horizon and show that it contains a universal saddle characterized by the surface gravity $2 \pi T_{\mathrm{H}}$. Our work demonstrates that the black hole horizon is the origin of the chaos, and suggests a close interplay between chaos and quark deconfinement.
\end{abstract}

DOI: 10.1103/PhysRevD.98.086007

\section{INTRODUCTION}

The renowned AdS/CFT correspondence [1] asserts that a certain class of quantum field theories allows a corresponding gravity description. Since classical gravity is characterized by its nonlinear solutions, black holes, characteristics of black hole horizons are believed to be a key to unveil the unknown mechanism of how the AdS/CFT works. In the course of research on shock waves near black hole horizons [2,3] (and subsequent study [4-8]), Maldacena, Shenker, and Stanford provided [9] a universal chaos bound for the Lyapunov exponent $\lambda$ of out-of-time-ordered (OTO) correlators $[3,10]$ in thermal quantum field theories,

$$
\lambda \leq \frac{2 \pi T}{\hbar}
$$

Here $T$ is the temperature, and in the gravity dual, it is the Hawking temperature of the black hole, equal to the surface gravity at the horizon.

The issue here is how the chaos of a fundamental string is derived near black hole horizons. The AdS/CFT correspondence is best understood in string theory, and the

Published by the American Physical Society under the terms of the Creative Commons Attribution 4.0 International license. Further distribution of this work must maintain attribution to the author(s) and the published article's title, journal citation, and DOI. Funded by SCOAP ${ }^{3}$. fundamental object in string theory is of course the fundamental string. In this paper we provide a concrete chaos analysis of motion of a fundamental string very near the black hole horizon.

Although chaos in string motion has been studied [11-21] in curved geometries, in view of the chaos bound (1), two important issues are left unsolved: First, the motion of the string needs to be near the black hole horizon, and second, the CFT interpretation of the string motion is indispensable. In this paper we address these two issues, by adopting a suspended string in the AdS black hole geometry.

One of the advantages to considering the suspended string is that it can probe near the black hole horizon in a natural way. In [22], a particle probing the region near the black hole horizon, pulled outward by some external force, was shown to have a universal behavior around the local maximum of the energy. The suspended string provides a natural setup for the universal chaos of the black hole horizon in AdS/CFT, by replacing the particle with a Nambu-Goto string, a natural object in the top-down AdS/CFT correspondence. In Refs. [23,24], the dynamics of the Nambu-Goto string in pure AdS background has been studied. For pure AdS, there is no sensitivity to initial conditions, although the energy cascade in the string worldsheet is observed. We expect that the effect of the event horizon on chaos can be extracted by the analysis of the suspended string dynamics in the black hole spacetime.

The other advantage is that the suspended string has a clear CFT interpretation [25-27]: a Wilson loop in thermal Yang-Mills theory, in other words, an interquark force. The 
dual CFT observable we calculate is the Lyapunov exponent $\lambda$ of chaos of the interquark force $\vec{F}$ :

$$
\langle\delta \vec{F}(t)\rangle \simeq e^{\lambda t}\langle\delta \vec{F}(0)\rangle
$$

where $\langle\delta \vec{F}(t)\rangle \equiv\langle\vec{F}(t)\rangle_{1}-\langle\vec{F}(t)\rangle_{2}$ is the difference of the interquark forces due to two different initial conditions indicated by \langle\rangle$_{1}$ and \langle\rangle$_{2}$ [the initial $(t \sim 0)$ infinitesimal kicks about the interquark distance $L(t)$ are chosen to be slightly different]. In the time evolution, the difference grows exponentially for chaotic systems, with the Lyapunov exponent $\lambda>0$.

Chaos of Wilson loops is important for understanding meson dynamics and deconfinement transition. The charmonium suppression in heavy ion collisions may be understood by an entropic interquark force [28-31], and the chaos could provide the entropy in a manner similar to [33-35]. At the deconfinement a turbulent behavior is expected [36,37], and the scaling of excited meson numbers [38-40] can be attributed to the chaos of mesons [41] and the chaos of the QCD string.

Let us briefly review the suspended string in AdS black hole geometry, with emphasis also on an unstable solution which probes the horizon. Consider a fundamental string suspended from the boundary of the AdS black hole geometry. The background metric in 10-dimensional spacetime is

$d s^{2}=\frac{r^{2}}{R^{2}}\left[-f(r) d t^{2}+d \vec{x}^{2}\right]+\frac{R^{2}}{r^{2} f(r)} d r^{2}+R^{2} d \Omega_{5}^{2}$

with $f(r) \equiv 1-r_{H}^{4} / r^{4}$, and $R$ is the AdS radius. The last term corresponds to the internal $S^{5}$ of the background geometry. The horizon is located at $r=r_{H}$, and the temperature of the black hole is given as $T_{H}=r_{H} / \pi R^{2}$. The action for a fundamental string in this AdS black hole geometry is simply given by

$$
S=-\mathcal{T} \int d \tau d \sigma \sqrt{-\operatorname{det}\left(G_{\mu \nu}[X] \partial_{\alpha} X^{\mu} \partial_{\beta} X^{\nu}\right)} .
$$

Here $\mu, \nu=0,1,2,3, r$ is the target space index and $\alpha$, $\beta=\tau, \sigma$ is the worldsheet index. We study the dynamics of the fundamental string in this geometry, in particular, when a part of the string is close to the black hole horizon $r=r_{H}$.

In the AdS/CFT correspondence, a string suspended from the boundary $r=\infty$ of the geometry corresponds to a pair of a quark and an anti-quark separated by the distance $L$ in the 4-dimensional CFT. The energy of the string is a quark-antiquark potential of $\mathcal{N}=4$ super Yang-Mills theory.

In Fig. 1, we show three possible shapes of the string for a fixed interquark separation $L$. The figure describes one

\footnotetext{
${ }^{1}$ Time evolution of the suspended string was analyzed in a different context; see [32].
}

half of the strings, since the shape is symmetric under a parity exchanging the quark and the antiquark. When $L$ is small (below some critical value), there are three possible configurations of the string: solid line, solid thick line, and dashed line in the figure. The dashed line is just a set of two parallel straight strings aligned along the $r$ direction, separated by the distance $L$, stuck to the black hole horizon. Solid lines are a suspended string whose asymptotic location is separated by the distance $L$. In particular, the solid thin line describes a local minimum of the energy, while the thick one is at a local maximum of the energy, for a given fixed $L$. For $L$ larger than a critical value, only the separate parallel string exists, and the interquark force disappears.

The goal of this paper is to calculate a Lyapunov exponent of a full numerical simulation of the suspended Nambu-Goto string. The suspended string has a clear interpretation in the CFT side: a Wilson loop. So the chaos described by the string is quantum chaos probed by the Wilson loop operator of non-Abelian gauge theories. In other words, the time evolution of the force between the quarks is exponentially sensitive to the difference of the initial conditions. Our calculation is a prediction for the time evolution of the interquark forces at finite temperature in $\mathcal{N}=4$ supersymmetric Yang-Mills (SYM) theory at large $N$ and in a strong coupling limit.

To study the origin of the chaos, we look at the solid thick line of the string in Fig. 1 since it probes very near the black hole horizon. This configuration has not been studied in the literature simply because it is not the minimum energy configuration. The unstable classical configuration of the string at the local maximum of the energy is provided

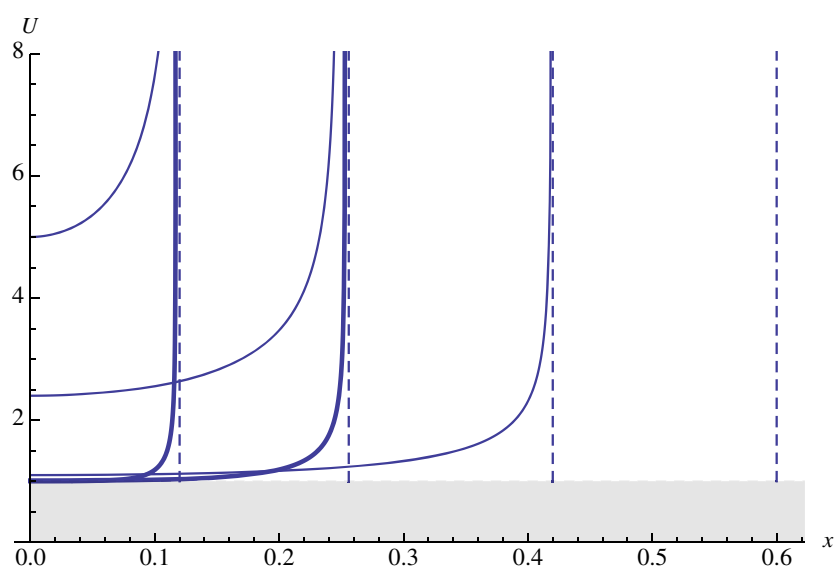

FIG. 1. The shape of a string suspended down to the nearhorizon region. We show the right half of the strings, for four choices of the asymptotic interquark separation $L$. Thin solid lines are connected strings at the local minima of the energy, and thick solid lines are connected strings at the local maxima of the energy. Dashed lines are strings stuck to the black hole horizon. The shaded region is the black hole, and the line $U \equiv r / r_{H}=1$ is the horizon. 
by the balance of the outward pulling force from the string tension and the gravity from the black hole, so it provides a natural realization of the particle model given in [22].

We provide three kinds of chaos analyses in this paper, and find a chaos nested near the black hole horizon. First, in Sec. II, we provide a toy model in which the suspended string is of a rectangular shape. It is natural to introduce such a toy model in view of the true shape of the solid thick lines in Fig. 1. The toy model shows the existence of the local maximum of the energy, at which the Lyapunov exponent of the chaos is evaluated to be universal and saturates the bound $\lambda=2 \pi T_{\mathrm{H}}$. In Appendix A we show that a more generic $(p, q)$ string in general near-horizon geometry of D3-F1-D1 bound states shows the same Lyapunov exponent; thus this value is universal.

Next, in Sec. III, we provide a chaos analysis of the fluctuation modes of the string. When the minimum energy configuration of the suspended string (the thin line in Fig. 1) fluctuates, the motion is expected to show chaos. We truncate the fluctuation to just the lowest and the first excited states, and introduce cubic order interaction calculated from the Nambu-Goto action; it is the minimal set of modes and interactions needed to show chaos. We find numerical evidence that chaos emerges due to the effect of the black hole horizon even in this simplified setup.

Finally, in Sec. IV, we provide a full nonlinear study of the Nambu-Goto string in the black hole background. With a "kick" at the boundary of the stable suspended string, the string starts to move in the curved geometry. We look at the time-dependent force which the quark feels, and also the motion of the bottom of the suspended string, and extract the Lyapunov exponent. We find that the measured Lyapunov exponent satisfies the universal bound, $\lambda \sim 0.065 \times 2 \pi T_{\mathrm{H}}$. This is our prediction for the chaos of the interquark forces in thermal gauge theories with an AdS-Schwarzschild gravity dual.

The last section is devoted to a summary and discussion of the interpretation of our Lyapunov exponents as a CFT out-of-time-order correlator.

\section{UNIVERSALITY IN A TOY STRING MODEL}

In this section, we present a string toy model which shows a universal chaos. The motion of the string is quite complicated since it is not integrable and is subject to a set of partial differential equations following from the action (4). To find a universal feature of the dynamics near the horizon, it is instructive to start with an approximation for the shape of the string. In view of Fig. 1, we are naturally led to consider a string with a rectangular shape (see Fig. 2) to study the motion of the string near the black hole horizon (see Fig. 2).

Suppose that the bottom part of the rectangle is at $r=r_{0}$, then the total action of the string is given by

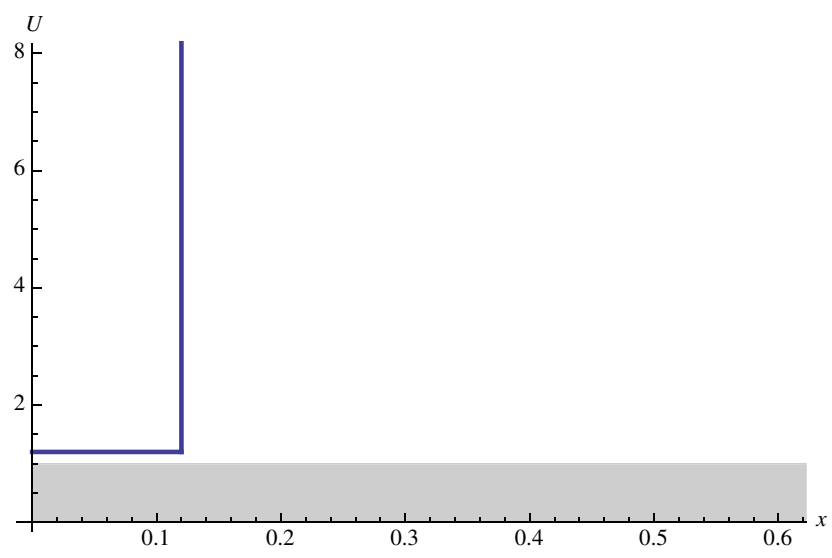

FIG. 2. The string with the rectangular shape studied in Sec. II.

$$
\begin{aligned}
S & =-\mathcal{T} \int d t\left[\sqrt{g_{00}\left(r_{0}\right) g_{x x}\left(r_{0}\right)} L-2 \int_{r_{H}}^{r_{0}} \sqrt{g_{00}(r) g_{r r}(r)} d r\right] \\
& =-\mathcal{T} \int d t\left[\frac{L}{R^{2}} \sqrt{r_{0}^{4}-r_{H}^{4}}-2\left(r_{0}-r_{H}\right)\right] .
\end{aligned}
$$

The first term is for the bottom part of the string, and the second term is the contribution from the vertical parts (the parallel two edges connecting $r=r_{0}$ and the AdS boundary). Note that the second term is the remainder after subtracting the infinite contribution $2 \int_{r_{0}}^{\infty} d r$, which is the length of the infinitely long strings, corresponding to the action of the two independent quarks.

The total energy of the string is then

$$
\mathcal{E}=\mathcal{T}\left[\frac{L}{R^{2}} \sqrt{r_{0}^{4}-r_{H}^{4}}-2\left(r_{0}-r_{H}\right)\right] .
$$

Extremizing this energy means

$$
r_{0}^{6}=\frac{R^{4}}{L^{2}}\left(r_{0}^{4}-r_{H}^{4}\right)
$$

This has two roots $r_{0}$ when $0<L / R^{2} r_{H}<(4 / 27)^{1 / 4}$, one of which corresponds to the local maximum of $\mathcal{E}$ near the black hole horizon and one which corresponds to the local minimum of $\mathcal{E}$ away from the horizon. We are interested in the case $r_{0}$ approaching the horizon, and in fact, for small $L$ this equation has a solution near the horizon. For small $L$, the solution is expanded as

$$
r_{0}^{\mathrm{sol}}=r_{H}\left(1+\frac{1}{4}\left(\frac{r_{H}}{R^{2}} L\right)^{2}+\frac{9}{32}\left(\frac{r_{H}}{R^{2}} L\right)^{4}+\cdots\right) .
$$

This solution actually maximizes the energy locally, since if we expand the energy for a small change of the location of the bottom of the string,

$$
r_{0}=r_{0}^{\mathrm{sol}}+\delta r
$$


then

$$
\mathcal{E}=\text { const }-\mathcal{T} \frac{2 R^{4}}{r_{H}^{3} L^{2}}(\delta r)^{2}+\cdots .
$$

So, the solution with $r_{0}=r_{0}^{\text {sol }}$ of (8) is at the top of the potential hill, maximizing the energy locally.

To look at the motion of the string, we evaluate the kinetic energy of the bottom part of the string moving slowly in the $r$ direction. When there is a time dependence $r_{0}(t)$, the relevant part of the action is

$$
S=-\mathcal{T} \int d t L \sqrt{-\left(g_{00}+g_{r r} \dot{r}_{0}^{2}\right) g_{x x}},
$$

which is expanded in terms of small $\dot{r}_{0}$ as

$$
S=-\mathcal{T} L \int d t \frac{r_{0}^{2}}{R^{2}} \sqrt{f\left(r_{0}\right)}\left(1-\frac{R^{4}}{2 r_{0}^{4} f\left(r_{0}\right)^{2}} \dot{r}_{0}^{2}+\cdots\right) .
$$

Evaluating the coefficient of the kinetic term $\dot{r}_{0}^{2}$ at $r_{0}^{\text {sol }}$ for small $L$, we obtain an effective action for the motion of the string, together with (10),

$$
S=\mathcal{T} \frac{R^{8}}{2 r_{H}^{5} L^{2}}\left(\frac{d}{d t} \delta r\right)^{2}+\mathcal{T} \frac{R^{4}}{r_{H}^{3} L^{2}}(\delta r)^{2} .
$$

Using the relation to the Hawking temperature of the black hole, $r_{H}=\pi R^{2} T_{\mathrm{H}}$, this effective action can be simply written as

$$
S=\mathcal{T} \frac{R^{8}}{2 r_{H}^{5} L^{2}}\left[\left(\frac{d}{d t} \delta r\right)^{2}+\left(2 \pi T_{\mathrm{H}}\right)^{2}(\delta r)^{2}\right] .
$$

Here, the factor $2 \pi T_{\mathrm{H}}$ is the surface gravity of the black hole. A generic solution of the equation of motion derived from the effective action (14) is given as

$$
\delta r=A \exp \left[2 \pi T_{\mathrm{H}} t\right]+B \exp \left[-2 \pi T_{\mathrm{H}} t\right]
$$

whose first term is exponentially growing. Interpreting the maximum point of the potential as a separatrix as in [22], we find that the Lyapunov exponent $\lambda$ is given by

$$
\lambda=2 \pi T_{\mathrm{H}}
$$

Therefore, the Lyapunov exponent is given by the Hawking temperature of the black hole. This saturates the bound conjectured in [9].

Note that the current system has a scaling symmetry: $(t, 1 / r, \vec{x}) \rightarrow c(t, 1 / r, \vec{x})$ and $r_{H} \rightarrow r_{H} / c$, where $c$ is a nonzero constant. Since the Lyapunov exponent scales as $\lambda \rightarrow \lambda / c$, the Lyapunov exponent must be proportional to $T_{\mathrm{H}}$. So, the coincidence with [9] should be understood as our nontrivial coefficient $2 \pi$. In Appendix A, however, we find that the universality of the Lyapunov exponent persists even when the fundamental string is generalized to the $(p, q)$ string in the near-horizon geometry of the D3-F1-D1 bound state at a finite temperature.

\section{CHAOS OF PERTURBATIVE STRING MOTION}

In the previous section, we argued that the Lyapunov exponent for a string moving in the near-horizon region takes a universal value that depends only on the horizon temperature. To make a firmer connection between this Lyapunov exponent and the chaotic behavior of the string, in the following sections we study the string motion and its chaotic behavior in more direct ways. In this section, we study an effective model for the perturbative string motion by truncating up to the first excited state. We also examine the full nonlinear dynamics of the string and evaluate the Lyapunov exponent in Sec. IV.

To describe the perturbative string motion, we follow the method of Ref. [42]. In this section, we take the unit of $R=r_{H}=1$.

We first study a static string configuration. Specifying the string location by $(r, x)=(r(\ell), x(\ell))$, where $\ell$ is the proper distance measured along the string from its tip, the action for the string becomes ${ }^{2}$

$$
S=-\mathcal{T} \int d \ell \sqrt{r^{\prime 2}(\ell)+\left(r^{4}-1\right) x^{\prime 2}(\ell)} .
$$

Since $\ell$ is the proper length along the string, the tangent vector along the string $t^{a}=\left(r^{\prime}(\ell), x^{\prime}(\ell)\right)$ becomes a unit vector, that is,

$$
\frac{r^{\prime 2}(\ell)}{r^{2} f(r)}+r^{2} f(r) x^{\prime 2}(\ell)=1 .
$$

Also, the action (17) implies an equation of motion for a static string given by

$$
\frac{d x}{d r}= \pm \sqrt{\frac{r_{0}^{4}-1}{\left(r^{4}-1\right)\left(r^{4}-r_{0}^{4}\right)}},
$$

where $r=r_{0}$ is the location of the tip of the string. Equations (18) and (19) fix the location of the static string $(r, x)=\left(r_{\mathrm{BG}}(\ell), x_{\mathrm{BG}}(\ell)\right)$, which will be used as the background solution to study small fluctuations around it. Below, we focus on the $x>0$ part of the string and choose the + sign in Eq. (19).

As explained in Sec. I, there is one stable and one unstable static configuration for the same boundary condition (the quark separation $L$ ). As the background solution, we take the unstable static string, which is constructed by setting $r_{0}$ sufficiently close to $r_{H}$. Below, we consider perturbative

\footnotetext{
${ }^{2} \mathrm{An}$ advantage of this parametrization is that the perturbation equations become regular everywhere on the string. This property helps the numerical integration to evaluate the truncated action (24).
} 


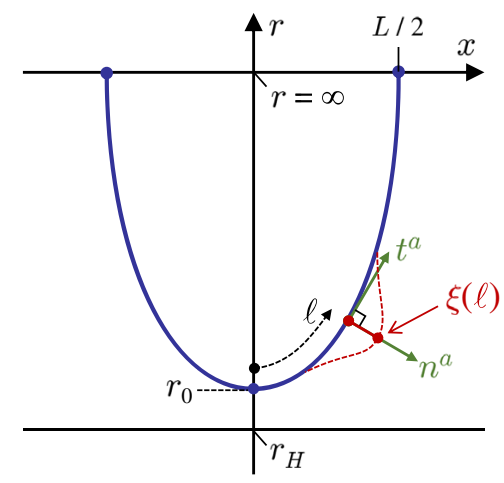

FIG. 3. Geometry of the perturbations around a static string.

motion around this unstable maximum taking into account the next-to-leading order perturbations. The leading-order action describes an oscillator with an unstable potential, and the next-to-leading order perturbation generates a trapping potential near the unstable equilibrium point. We consider the string motion in this trapping potential in the following analysis. An advantage of this approach is that the potential for the string motion is well approximated near the unstable maximum, which is expected to give a dominant contribution to the chaotic behavior of the string as observed in Sec. II and Ref. [22], though the approximation may not be accurate near the stable equilibrium point of the potential.

To describe perturbations around the static string, we introduce the unit normal on the string by

$$
n_{a}=\frac{r_{\mathrm{BG}}^{2}(\ell)}{\sqrt{r_{\mathrm{BG}}^{4}(\ell)-1}}\left(-x_{\mathrm{BG}}^{\prime}(\ell), r_{\mathrm{BG}}^{\prime}(\ell)\right) \text {. }
$$

We perturb the string toward the normal direction $n^{a}$ by a proper distance $\xi(t, \ell)$. Then the target space coordinate of the perturbed string is given by (see Fig. 3)

$r=r_{\mathrm{BG}}(\ell)+\xi(t, \ell) n^{r}(\ell), \quad x=x_{\mathrm{BG}}(\ell)+\xi(t, \ell) n^{x}(\ell)$.

Expanding the action (4) up to the third order in $\xi(t, \ell)$, we can construct an effective action for the perturbative string motion. We defer the derivation to Appendix B and summarize only the construction procedure and the final result below. The perturbation equation obtained from the quadratic action is given by

$\omega^{2} C_{t t}(\ell) \xi(\ell)+\partial_{\ell}\left[C_{\ell \ell}(\ell) \xi^{\prime}(\ell)\right]-C_{00}(\ell) \xi(\ell)=0$,

where the coefficients $C_{t t}, C_{\ell \ell}, C_{00}$ are given by Eq. (B5), and we separated the variable as $\xi(t, \ell)=\xi(\ell) e^{i \omega t}$. Solutions to this equation can be expanded using the eigenfunctions. In our analysis, we truncate the solution up to the first two excitations as

$$
\xi(t, \ell)=c_{0}(t) e_{0}(\ell)+c_{1}(t) e_{1}(\ell)
$$

where $e_{0,1}(\ell)$ are the normalized mode functions with the lowest and the next lowest eigenfrequencies. Next, we evaluate the cubic-order part of the action using the truncated solution (23), which results in

$$
\begin{aligned}
\frac{S^{(3)}}{\mathcal{T}} & =\int d t \int_{-\infty}^{\infty} d \ell\left\{\left[\left(C_{0}-\frac{C_{1}^{\prime}}{3}\right) e_{0}^{3}+C_{2} e_{0} e_{0}^{\prime}\right] c_{0}^{3}(t)\right. \\
& +\left[3\left(C_{0}-\frac{C_{1}^{\prime}}{3}\right) e_{0} e_{1}^{2}+C_{2}\left(e_{0} e_{1}^{\prime 2}+2 e_{0}^{\prime} e_{1} e_{1}^{\prime}\right)\right] c_{0}(t) c_{1}^{2}(t) \\
& \left.+C_{3} e_{0}^{3} c_{0} \dot{c}_{0}^{2}+C_{3} e_{0} e_{1}^{2} c_{0} \dot{c}_{1}^{2}+2 C_{3} e_{0} e_{1}^{2} \dot{c}_{0} c_{1} \dot{c}_{1}\right\}
\end{aligned}
$$

where $C_{0,1,2,3}(\ell)$ are functions of the background solution $r_{\mathrm{BG}}(\ell)$. Evaluating the action (24) numerically we can construct the cubic action. For example, when the tip of the string is at $r=r_{0}=1.1$, the total perturbative action is given by

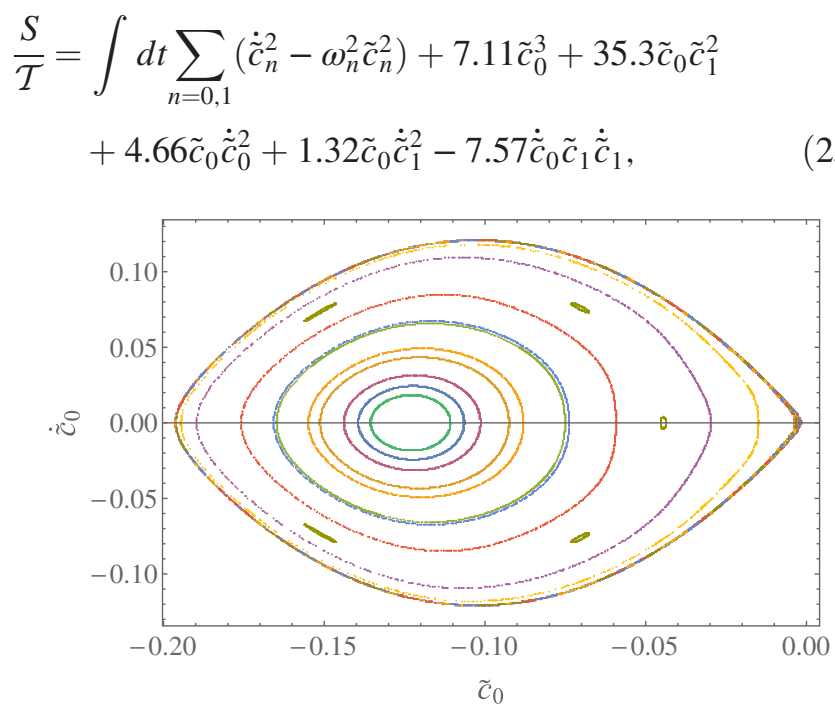

(a) Poincaré plot

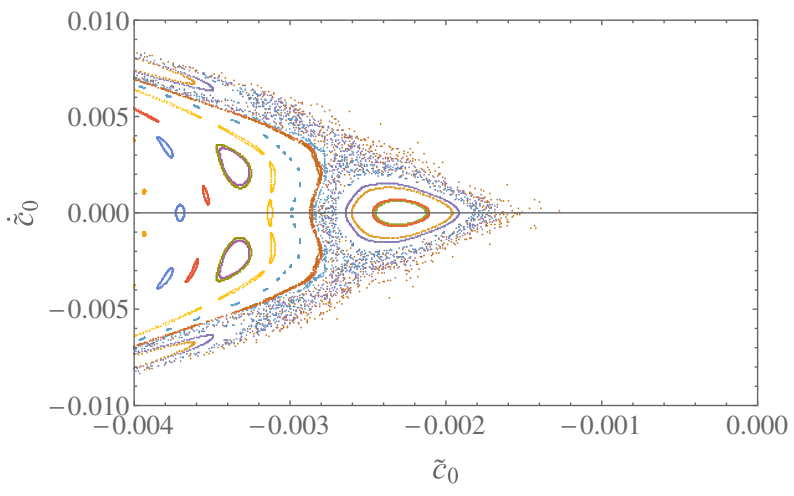

(b) Closeup of Fig. 4(a)

FIG. 4. Poincaré plot obtained from the action (25) for orbits with energy $E=9.28 \times 10^{-6}$ and $0<t<8000$. Though most of the orbits form regular tori, some orbits near the saddle point of the potential form a scattered plot indicating chaos. 
where $\omega_{0}^{2}=-1.40$ and $\omega_{1}^{2}=7.57$, and $\tilde{c}_{0,1}(t)$ are functions of $c_{0,1}(t)$ introduced by Eq. (B13) to stabilize the time evolution.

Solving the time evolution based on the action (25), we can examine if the system exhibits chaos or not by constructing a Poincare section. In Fig. 4, we show the Poincaré sections defined by $\tilde{c}_{1}(t)=0$ and $\dot{\tilde{c}}_{1}(t)>0$ for bound orbits within the trapping potential. From the action (25) we can check that the energy of a bound orbit is negative, since the value of the potential at the unstable saddle point is equal to zero. In Fig. 4, we show the Poincaré plot for the orbits with energy $E=9.28 \times 10^{-6}$ and $0<t<8000$, where points with different colors correspond to the numerical data of orbits for different initial data. Most of the orbits form regular tori, while we can also see that some tori near the saddle point of the potential collapse to become scattered plots. This saddle point corresponds to the black hole horizon; hence we confirm that a black hole horizon works as a source of chaos. Evaluating the Lyapunov exponent for these chaotic orbits, we find $\lambda \simeq 0.04 \times 2 \pi T_{H}$, which satisfies the bound (1). In Sec. IV we study fully nonlinear motion of the string and find that its Lyapunov exponent is of the same order as this value.

\section{CHAOS OF NONLINEAR DYNAMICS OF A STRING}

In this section, we demonstrate chaos of the string by full numerical simulations to examine if the Lyapunov exponent of the chaotic motion obeys the bound (1). For this purpose we employ the numerical method to study a dynamical string in AdS developed in Refs. [23,24]. We take the static stable string configuration as the initial condition of the time evolution, and to induce the nonlinear dynamics of the string, we "quench" the position of the string endpoints on the AdS boundary. Holographically, it would correspond to the nonlinear dynamics of the gluon flux tube induced by the motion of the quark-antiquark pair.

\section{A. Basic equations}

To facilitate the numerical calculation, we introduce the ingoing Eddington-Finkelstein coordinates, which are regular on the black hole horizon. In these coordinates the metric (3) is given by

$$
d s^{2}=\frac{R^{2}}{z^{2}}\left[-f(z) d V^{2}-2 d V d z+d \vec{x}^{2}\right]+R^{2} d \Omega_{5}^{2},
$$

where $z=R^{2} / r, \quad z_{H}=R^{2} / r_{H}, f(z)=1-z^{4} / z_{H}^{4}$ and $V=t+z_{*}(z)$. Here, we introduce the tortoise coordinate $z_{*}(z)=-\int_{0}^{z} d z^{\prime} / f\left(z^{\prime}\right)$. As the worldsheet coordinates, we take double null coordinates $(u, v)$ and specify the string position as $V=V(u, v), z=Z(u, v), \vec{x}=\vec{X}(u, v)$. The condition on the induced metric $h_{a b}$ for $u, v$ to be null coordinates is given by $h_{u u}=h_{v v}=0$. In the double null coordinates, the Lagrangian for the string motion is proportional to $h_{u v}$, and the Nambu-Goto action (4) becomes

$$
\begin{aligned}
S= & -R^{2} \mathcal{T} \int d u d v \frac{1}{Z^{2}} \\
& \times\left[-f(Z) V_{, u} V_{, v}-V_{, u} Z_{, v}-V_{, v} Z_{, u}+\vec{X}_{, u} \cdot \vec{X}_{, v}\right] .
\end{aligned}
$$

From the action, we obtain the evolution equations of the string as

$$
\begin{aligned}
V_{, u v}= & -\left(\frac{f}{Z}-\frac{f^{\prime}}{2}\right) V_{, u} V_{, v}+\frac{1}{Z} \vec{X}_{, u} \cdot \vec{X}_{, v}, \\
Z_{, u v}= & \left(\frac{f}{Z}-\frac{f^{\prime}}{2}\right)\left(f V_{, u} V_{, v}+V_{, u} Z_{, v}+V_{, v} Z_{, u}\right) \\
& +\frac{2}{Z} Z_{, u} Z_{, v}-\frac{f}{Z} \vec{X}_{, u} \cdot \vec{X}_{, v}, \\
\vec{X}_{, u v}= & \frac{1}{Z}\left(\vec{X}_{, u} Z_{, v}+\vec{X}_{, v} Z_{, u}\right),
\end{aligned}
$$

where $f^{\prime}=d f / d z$. To simulate the time evolution of the string, we solve these partial differential equations numerically. We follow the numerical method developed in Refs. [23,24] in which the double null formalism is employed. We take the uniform mesh along the $u$ and $v$ directions. The number of grids along the $u$ direction is $N=2^{10}$ in this paper. The same mesh size is also used for the $v$ direction. For a check of the numerical accuracy, we monitor the violation of constraints $h_{u u}=h_{v v}=0$ throughout our numerical calculations. We find that the violation is on the order of $10^{-3}$ for $N=2^{10}$ compared to the typical scale of the constraints.

\section{B. Boundary conditions}

Hereafter, we take the unit $z_{H}=1$, in which the Hawking temperature is $T_{H}=1 / \pi$. [Because the AdS scale $R$ appears just as a factor of the metric (26), its value does not effect the string dynamics.] Using the residual coordinate freedom, $u \rightarrow F(u)$ and $v \rightarrow G(v)$, we put boundaries of the worldsheet at $u-v= \pm \pi / 2$. As the initial condition, we use a static string shown in Fig. 1. For fixed $z_{H}=1$, the series of static solutions can be regarded as a one-parameter family of $z_{\text {center }}$, where $z_{\text {center }}$ represents the $z$-coordinate at the tip of the static string. We use the static solution with $z_{\text {center }}=0.5$ as initial data. Then, the separation of string endpoints is given by $L=0.591$.

To induce the nonlinear motion of the string, we impose time-dependent boundary conditions for the string endpoints. Introducing time and spatial coordinates on the worldsheet as $\tau=u+v$ and $\sigma=u-v$, we consider 


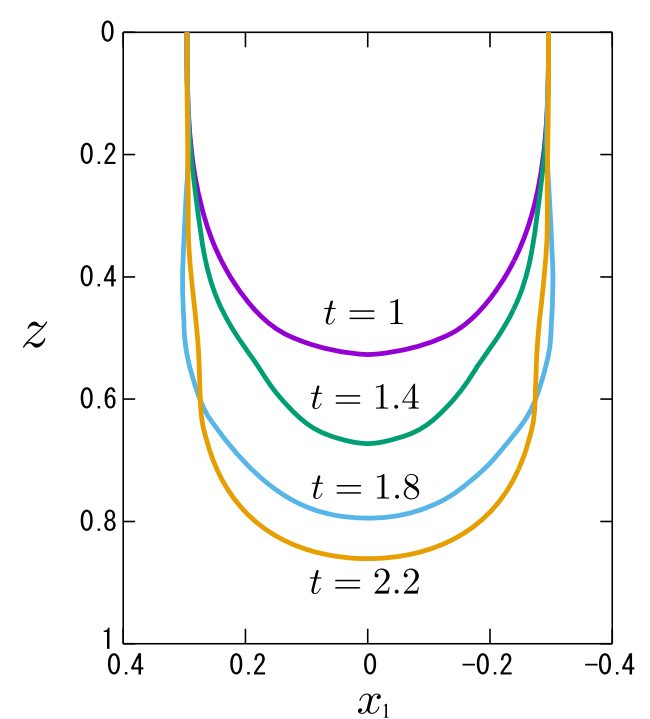

(a) Early time evolution

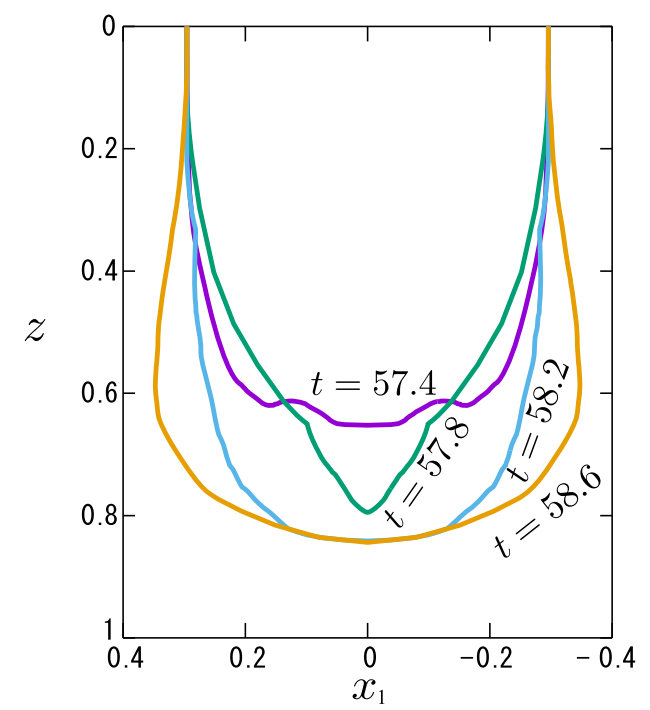

(b) Late time evolution

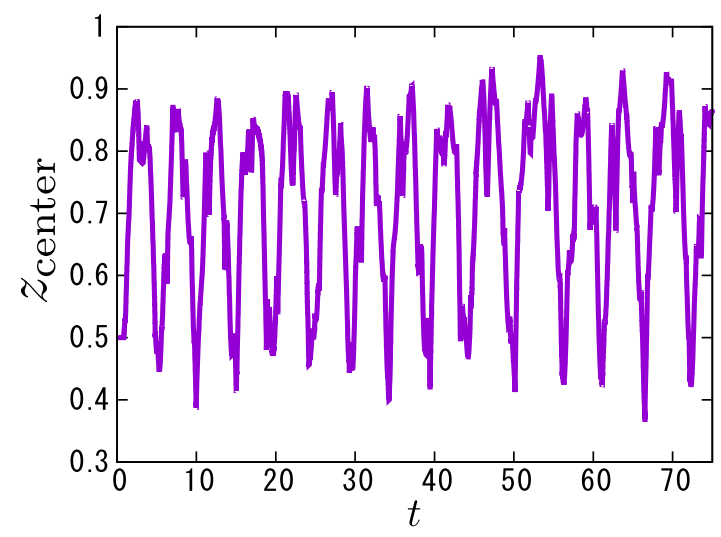

(c) $z$-coordinate at center

FIG. 5. Dynamical string for $\epsilon=0.030013$ and $\Delta \tau=2$. In panels (a) and (b), the motion in the $x_{2,3}$ directions is not shown. Panel (c) shows the time evolution of the location of the string on the symmetry axis $x_{1}=0, z_{\text {center }}=Z(\tau, \sigma=0)$, with respect to the bulk time coordinate $t$. the quench of positions of the string endpoints along the transverse direction ${ }^{3}$ :

$$
X_{2}(\tau, \sigma= \pm \pi / 2)=\epsilon \alpha(\tau ; \Delta \tau),
$$

where

$$
\alpha(\tau ; \Delta \tau)=\left\{\begin{array}{ll}
\exp \left[2\left(4-\frac{\Delta \tau}{\tau}-\frac{\Delta \tau}{\Delta \tau-\tau}\right)\right] & (0<\tau<\Delta \tau) \\
0 & (\text { else })
\end{array} .\right.
$$

One can check that this function is $C^{\infty}$ and has a compact support in $0 \leq \tau \leq \Delta \tau$. The two parameters $\epsilon$ and $\Delta \tau$ represent the amplitude and timescale of the quench. Boundary conditions for the other variables are $X_{1}(\tau, \sigma= \pm \pi / 2)= \pm L / 2, \quad X_{3}(\tau, \sigma= \pm \pi / 2)=0 \quad$ and $Z(\tau, \sigma= \pm \pi / 2)=0$, and $V(\tau, \sigma= \pm \pi / 2)$ are determined by the constraints $h_{u u}=h_{v v}=0$. For these boundary conditions, the string motion is symmetric under $x \rightarrow-x$.

\section{Time evolution of the string}

Figure 5 shows the time evolution of the string in the $(x, z)$-plane for $\epsilon=0.030013$ and $\Delta \tau=2$. In this figure, we take the constant time slice in terms of the bulk coordinate $t$ defined by

$$
t=V(u, v)+z_{*}(Z(u, v)) .
$$

Panels (a) and (b) are snapshots of the string for early times $(1 \leq t \leq 2.2)$ and late times $(57.4 \leq t \leq 58.6)$. (Although the string is also moving along the $x_{2}$ direction, we do not show it for visibility.) At early times, the string profile seems smooth. On the other hand, at late times, we can observe small spatial fluctuations. This would reflect the energy cascade on the string worldsheet in nonintegrable geometries [21]. Panel (c) shows the time dependence of the $z$-coordinate at the tip of the string, $z_{\text {center }} \equiv Z(\tau, \sigma=0)$, with respect to the bulk time coordinate $t$ at the tip. To check the numerical accuracy, we monitor the violation of constraints $h_{u u}=h_{v v}=0$. The violation is $\sim 10^{-3}$ compared to the typical scale of the dynamical solution.

\section{Growth of perturbations}

To examine the sensitivity of the string motion to the initial conditions, we consider the linear perturbation $V \rightarrow V+\delta V, Z \rightarrow Z+\delta Z$ and $\vec{X} \rightarrow \vec{X}+\delta \vec{X}$. We numerically solve the linear equations for $(\delta V, \delta Z, \delta \vec{X})$ on the time-dependent background. Initial conditions are zero for all variables. Boundary conditions are $\delta X_{2}(\tau, \sigma= \pm \pi / 2)=$ $\alpha(\tau ; \Delta \tau=2)$ and $\left.\left(\delta X_{1}, \delta X_{3}, \delta Z\right)\right|_{\sigma= \pm \pi / 2}=0$. Again, $\delta V(\tau, \sigma= \pm \pi / 2)$ are determined by linearized constraints $\delta h_{u u}=\delta h_{v v}=0$.

\footnotetext{
${ }^{3}$ For $X_{2}$-quench, the violation of constraints $h_{u u}=h_{v v}=0$ is smaller than that of $X_{1}$-quench. Thus, we take $X_{2}$ as the quench direction for sufficiently long time evolution, enough to read out the Lyapunov exponent.
} 


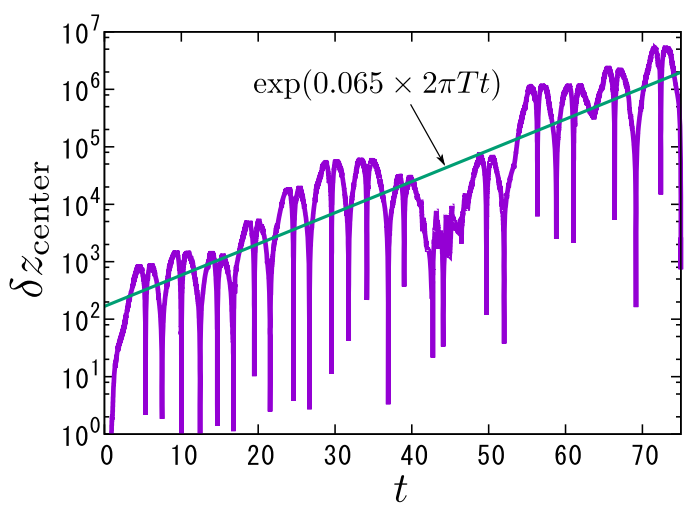

(a) $\epsilon=0.030013$

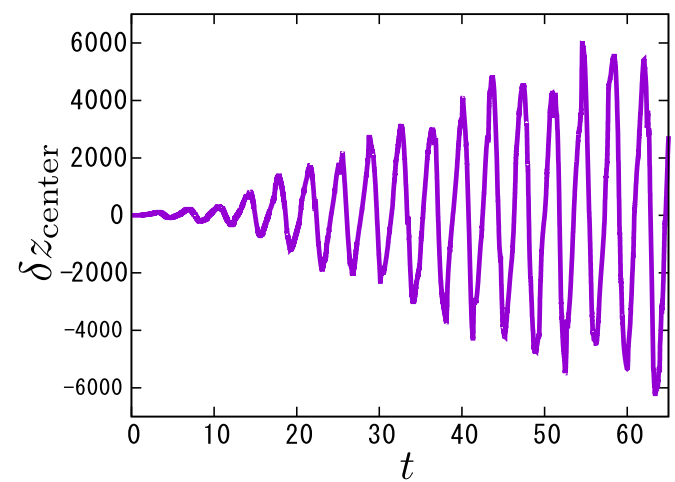

(b) $\epsilon=0.029$

FIG. 6. Sensitivity of the string tip position $\delta z_{\text {center }}$ to initial conditions.

The results of the time evolution of the linear perturbation are summarized as follows. Figure 6(a) shows the time evolution of $\delta Z$ at the center of the string for $\epsilon=0.030013$. As the horizontal axis, we take the bulk time coordinate $t=V(\tau, \sigma= \pm \pi / 2)+z_{*}(Z(\tau, \sigma= \pm \pi / 2))$. We can see an exponential growth of the oscillation amplitude that demonstrates chaos. Fitting the amplitude, we obtain the positive Lyapunov exponent as $\lambda \simeq 0.125=0.0625 \times 2 \pi T_{H}$. This is smaller than the Lyapunov bound $2 \pi T_{H}$.

The Lyapunov exponent can also be measured from an observable of the boundary field theory. Expanding the dynamical string solution $\vec{X}(t, z)$ near the AdS boundary with respect to $z$, we have the expectation value of the force as the coefficient of $z^{3}$, which is the conjugate operator of the quark position. As shown in Ref. [23], when the string endpoint is not moving, the force acting on the quark is given by

$$
\langle\vec{F}(t)\rangle=\left.\frac{\sqrt{\lambda_{\text {t Hooft }}}}{4 \pi} \partial_{z}^{3} \vec{X}\right|_{z=0},
$$

where $\lambda_{\text {t Hooft }}=R^{4} / \alpha^{\prime 2}$ is the 't Hooft coupling. Figure 7 shows the perturbation of the force $\langle\delta \vec{F}\rangle$. Again, we take the bulk time coordinate for the horizontal axis. We have dropped $\sqrt{\lambda_{\text {t Hooft }}}$ in this plot. Note that $\langle\delta \vec{F}\rangle$ grows

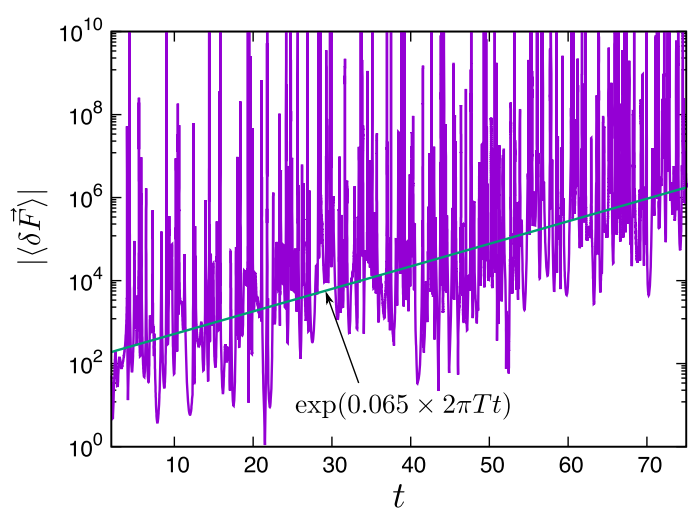

FIG. 7. Sensitivity of the force acting on the quark in the boundary field theory.

exponentially, and its Lyapunov exponent is consistent with that from $\delta z_{\text {center }}(t)$. We find chaos of the interquark forces, and it is a holographic prediction for the force in thermal gauge theories with the AdS-Schwarzschild gravity dual.

Some comments about the numerical simulations are in order. We see that $\langle\delta \vec{F}\rangle$ is spikier than $\delta z_{\text {center }}$ as a function of $t$. This spiky profile is caused by cusps created on the string. In Ref. [23], it is shown that the force on the quark diverges when the cusp arrives at the AdS boundary.

We can see the chaotic behavior only in a small region of the parameter space of $\epsilon$, as shown in Fig. 6(b). In this figure, the amplitude of the quench is slightly decreased to $\epsilon=0.029$. Then we see that the amplitude ceases to grow exponentially. [Note that the scale of the vertical axis in Fig. 6(b) is linear while that in Fig. 6(a) is log.] On the other hand, if we increase $\epsilon$ to $\epsilon \gtrsim 0.03002$, the string plunges into the black hole at finite time.

\section{SUMMARY AND DISCUSSIONS}

In this paper, we performed a nonlinear numerical simulation of time evolution of a suspended Nambu-Goto string in the AdS-Schwarzschild background. In Sec. IV we discovered that the force measured at the asymptotic boundary of the string has an exponential sensitivity to small differences in the initial conditions. We conclude that the quark-antiquark force is chaotic in thermal gauge theories.

A perturbative analysis given in Sec. III shows the collapse of regular tori of phase space orbits and also a positive Lyapunov exponent, as expected. The origin of chaos should be the black hole horizon, and in Sec. II our toy string model finds an unstable saddle near the horizon, characterized universally by the surface gravity $2 \pi T_{\mathrm{H}}$.

The important point in our work is that the chaotic string motion in the bulk has a definite CFT interpretation: the Wilson loop, or equivalently, the interquark forces. The exponential growth is physically provided by (2) with the Lyapunov exponent $\lambda$. The holography calculates $\langle F(t)\rangle$ at large $N_{c}$ and at a strong coupling limit, and the measured Lyapunov exponent is shown to satisfy the 
universal chaos bound (1), as seen in Sec. IV. This is our holographic prediction for thermal gauge theories.

The chaos near the black hole horizon has often been discussed in terms of the OTO correlators, and here we provide a relation to them. We expect that the exponential growth would be calculated by the following OTO correlator in the $\mathcal{N}=4 \mathrm{SYM}$,

$$
C_{T}(t) \equiv\left\langle\operatorname{Ptr} \exp \left[i \oint A_{\mu} d x^{\mu}\right] W(t) F_{01}(0) W(t) F_{01}(0)\right\rangle_{T}
$$

This is an OTO correlator in the SYM, where the ordering of the operators $W(t)$ and $F_{01}(0)$ is not time-ordered. The first part is the standard Wilson loop operator with a rectangular shape (the spatial separation is $L$ along the $x^{1}$ direction while the time length is $\left.T \rightarrow \infty\right)$. Here $W(t)$ is a gauge-invariant operator inserted at $t=0$, which could be, e.g., a spatial integration of the energy momentum tensor, $\int d^{3} x T_{00}(t)$. The field strength $F_{01}(t=0)$, or a plaquette, is inserted on the path of the Wilson loop at $t=0$, and basically it amounts to an infinitesimal deformation of the path of the Wilson loop at the location.

The reason why we expect that $C_{T}(t)$ in (33) can detect the exponential growth of the unstable string fluctuation is as follows. In [3] and the subsequent study, the OTO correlation function of the form $\langle W(t) V(0) W(t) V(0)\rangle_{T}$ was shown to possess an exponential growth in time, where $W(t)$ is an operator perturbing the system while $V(0)$ is another operator for which one wants to evaluate the correlation function. In the gravity dual, the operator $W$ perturbs the background geometry with a horizon. A timeordered correlation function should approach a factorized $\langle W(t) W(t)\rangle\langle V(0) V(0)\rangle$, while the OTO correlation function should decay with the Lyapunov exponent. In our case the operator $V$ should correspond to an infinitesimal fluctuation of the Wilson loop, as the Wilson loop itself is a background on which the correlation function is measured. And the operator $W$ should be the one perturbing the system, so it can be inserted anywhere. In the gravity dual, we have found that the Wilson loop fluctuation can have an exponential growth near the horizon, thus a natural OTO correlator, which we can suggest is (33). ${ }^{4}$

The unstable string configuration plays the role of instanton amplitudes in string breaking. The unstable configuration is a saddle point dividing the connected and broken string configurations; thus the Lyapunov exponent is expected to be relevant to the instanton amplitude at the string breaking transition. The universality

\footnotetext{
${ }^{4}$ Note that the OTOC defined in [9] uses a peculiar insertion of divided thermal ensemble operators $\exp [-\beta H / 4]$; thus our chaotic $\langle\delta F(t)\rangle$ may not be written in the form of OTOC defined in [9].
}

seen in this paper is important in showing that the breaking amplitude in the WKB approximation should be given by

$$
\Gamma \sim \exp [-c L T]
$$

with some positive numerical coefficient $c$, since we can only use the temperature for fitting the dimension of the exponent. The coefficient $c$ can be calculated using our Lyapunov exponent.

The string breaking is known to be associated with thermal entropy production [28-31], which is argued to be relevant for quarkonium dissociation. On the other hand, the Lyapunov exponent provides a Kolmogorov-Sinai entropy production rate, and we expect that these two issues are related to each other. It may be interesting also to examine the connection of this string breaking phenomenon to the imaginary part of the interquark potential [43-45] and the meson melting phenomenon in holography [46].

\section{ACKNOWLEDGMENTS}

K. H. would like to thank T. Hatsuda, Y. Hidaka, and M. Hongo for valuable comments. The work of K.H. was supported in part by JSPS KAKENHI Grants No. JP15H03658, No. JP15K13483, and No. JP17H06462. The work of N. T. was supported in part by JSPS KAKENHI Grant No. 16H06932.

\section{APPENDIX A: UNIVERSALITY IN A $(p, q)$ STRING}

In Sec. II, we observed that the Lyapunov exponent for a fundamental string takes a universal value that depends only on the horizon temperature but is independent of the other physical parameters. In this appendix, let us consider a more generic situation in string theory to examine the universality of the Lyapunov exponent.

We consider a $(p, q)$ string in the near-horizon geometry of the D3-F1-D1 bound state with temperature. For the action of the $(p, q)$-string action, we use the S-duality covariant action [47]

$$
\begin{aligned}
S= & -\int d t d \sigma\left[\sqrt{q^{2} e^{-2 \phi}+(p-q \chi)^{2}} \sqrt{-\operatorname{det} G[X]}\right. \\
& \left.+\left(q A_{t \mu}+p B_{t \mu}\right) \frac{d X^{\mu}}{d \sigma}\right] .
\end{aligned}
$$

Here $A$ and $B$ are the Ramond-Ramond (RR) and NS-NS 2form, and $\phi$ and $\chi$ are the dilaton and the RR 0 -form axion, respectively. The near-horizon geometry of the D3-F1-D1 bound state is given in [48],

$$
\begin{gathered}
d s^{2}=F^{-1 / 2}\left(h^{\prime}\left(-f d t^{2}+d x_{1}^{2}\right)+h\left(d x_{2}^{2}+d x_{3}^{2}\right)\right) \\
+F^{1 / 2} f^{-1} d r^{2}, \\
c \\
e^{2 \phi}=g_{s}^{2} h h^{\prime},
\end{gathered}
$$




$$
\begin{gathered}
\chi=\frac{-1}{g_{s} F} \tan \varphi \sin \theta, \\
B_{01}=H^{-1} \operatorname{coth} \alpha \sin \varphi, \\
A_{01}=-H^{-1} \operatorname{coth} \alpha \sin \varphi,
\end{gathered}
$$

with the following definitions of the functions,

$$
\begin{aligned}
f & \equiv 1-\frac{r_{H}^{4}}{r^{4}}, \quad H \equiv \frac{r_{H}^{4} \sinh ^{4} \alpha}{r^{4}}, \quad h \equiv F / G \\
h^{\prime} & \equiv F / H, \quad F \equiv 1+\cos ^{2} \varphi \frac{r_{H}^{4}}{\sinh ^{2} \alpha} r^{4} \\
G & \equiv 1+\cos ^{2} \varphi \cos ^{2} \theta \frac{r_{H}^{4} \sinh ^{2} \alpha}{r^{4}} .
\end{aligned}
$$

The background geometry is quite involved, but after substituting the background into the $(p, q)$-string action, we obtain, for the rectangular shape,

$$
\begin{aligned}
S= & -\int d t\left[L c_{1} \sqrt{r_{0}-r_{H}} \sqrt{1-\frac{c_{2}}{\left(r_{0}-r_{H}\right)^{2}} \dot{r}_{0}^{2}}\right. \\
& \left.-\left(c_{3}+c_{4} L\right)\left(r_{0}-r_{H}\right)\right] .
\end{aligned}
$$

Here, $c_{i}(i=1,2,3,4)$ is a constant $\left(c_{1}, c_{2}\right.$ and $c_{3}$ are positive). It is a function of the parameters of the background geometry, $\left(\alpha, \varphi, \theta, g_{2}\right)$, and also of $(p, q)$. With this action, we find the maximum of the potential energy. As before, we expand the action around that point and also expand it to the leading order in the kinetic term; then we obtain

$$
S=\frac{-c_{1}^{2} L^{2}}{4\left(c_{3}+c_{4} L\right)}+\frac{4 c_{2}\left(c_{3}+c_{4} L\right)^{3}}{c_{1}^{2} L^{2}}\left[\left(\frac{d}{d t} \delta r\right)^{2}+\frac{1}{4 c_{2}}(\delta r)^{2}\right] .
$$

Let us evaluate the coefficient $c_{2}$ explicitly. It is given by

$$
\frac{c_{2}}{\left(r_{0}-r_{H}\right)^{2}}=\frac{F}{h^{\prime} f^{2}}
$$

and at the near-horizon region $r_{0} \sim r_{H}$, we find

$$
c_{2}=\frac{r_{H}^{2} \cosh ^{2} \alpha}{16} \text {. }
$$

The corrections, which are of order $\mathcal{O}(L)$, can be ignored for our interest of small L. Noting that the Hawking temperature of this generic geometry is given by

$$
T_{\mathrm{H}}=\frac{1}{\pi r_{H} \cosh \alpha},
$$

we find that the action at the top of the potential hill for slow motion of the rectangular $(p, q)$ string is

$$
S=\frac{4 c_{2}\left(c_{3}+c_{4} L\right)^{3}}{c_{1}^{2} L^{2}}\left[\left(\frac{d}{d t} \delta r\right)^{2}+\left(2 \pi T_{\mathrm{H}}\right)^{2}(\delta r)^{2}\right] .
$$

This has the same form as the previous case (14). The dynamics is completely governed by the Hawking temperature, again. So the Lyapunov exponent $\lambda$ for the motion of the $(p, q)$-string in the near-horizon geometry of the D3-D1-F1 bound state background is given again by

$$
\lambda=2 \pi T_{\mathrm{H}}
$$

Hence the value of the Lyapunov exponent is universal and is given by the Hawking temperature, even in this generalized setup.

\section{APPENDIX B: DERIVATION OF EFFECTIVE ACTION}

In this appendix, we show the details of the derivation of the perturbative action (25) studied in Sec. III. As explained there, we parametrize the perturbation of a static string using the proper distance along the normal vector of the string trajectory defined by Eqs. (20) and (21) and Fig. 3.

The action for the perturbative motion is derived by expanding the metric functions as

$$
\begin{aligned}
g_{\mu \nu}(r)= & g_{\mu \nu}\left(r_{\mathrm{BG}}\right)+g_{\mu \nu}^{\prime}\left(r_{\mathrm{BG}}\right) \xi n^{r} \\
& +\frac{g_{\mu \nu}^{\prime \prime}\left(r_{\mathrm{BG}}\right)}{2}\left(\xi n^{r}\right)^{2}+\frac{g_{\mu \nu}^{\prime \prime \prime}\left(r_{\mathrm{BG}}\right)}{6}\left(\xi n^{r}\right)^{3}+\cdots
\end{aligned}
$$

and using the line elements given by

$$
\begin{aligned}
& d x=\left(x_{\mathrm{BG}}^{\prime}+\left(n^{x}\right)^{\prime} \xi+n^{x} \xi^{\prime}\right) d \ell+n^{x} \dot{\xi} d t, \\
& d r=\left(r_{\mathrm{BG}}^{\prime}+\left(n^{r}\right)^{\prime} \xi+n^{r} \xi^{\prime}\right) d \ell+n^{r} \dot{\xi} d t,
\end{aligned}
$$

where a dot denotes a $t$ derivative.

Up to a surface term, the quadratic action is given by

$\frac{S^{(2)}}{\mathcal{T}}=\int d t \int_{-\infty}^{\infty} d \ell\left[C_{t t}(\ell) \dot{\xi}^{2}-C_{\ell \ell}(\ell) \xi^{\prime 2}-C_{00}(\ell) \xi^{2}\right]$

where

$$
\begin{aligned}
C_{t t}(\ell) & =\frac{r_{\mathrm{BG}}(\ell)}{2 \sqrt{r_{\mathrm{BG}}^{4}(\ell)-1}}, \quad C_{\ell \ell}(\ell)=\frac{\sqrt{r_{\mathrm{BG}}^{4}(\ell)-1}}{2 r_{\mathrm{BG}}(\ell)}, \\
C_{00}(\ell) & =\frac{r_{0}^{4}+3 r_{\mathrm{BG}}^{4}(\ell)-4 r_{0}^{4} r_{\mathrm{BG}}^{4}(\ell)-r_{0}^{4} r_{\mathrm{BG}}^{8}(\ell)+r_{\mathrm{BG}}^{12}(\ell)}{r_{\mathrm{BG}}^{5}(\ell)\left(r_{\mathrm{BG}}^{4}(\ell)-1\right)^{3 / 2}} .
\end{aligned}
$$


Then the perturbation equation is given by

$\omega^{2} C_{t t}(\ell) \xi(\ell)+\partial_{\ell}\left[C_{\ell \ell}(\ell) \xi^{\prime}(\ell)\right]-C_{00}(\ell) \xi(\ell)=0$,

where we separated the variable as $\xi(t, \ell)=\xi(\ell) e^{i \omega t}$. This equation is of the Sturm-Liouville form with the weight function $W(\ell)=C_{t t}(\ell)$, with which the inner product is defined as

$$
(\xi, \zeta) \equiv \int_{-\infty}^{\infty} W(\ell) \xi(\ell) \zeta(\ell) d \ell .
$$

Then we truncate the perturbation up to the first two lowest excitations as

$$
\xi=c_{0}(t) e_{0}(\ell)+c_{1}(t) e_{1}(\ell), \quad\left(e_{m}, e_{n}\right)=\delta_{m n},
$$

where $e_{0,1}(\ell)$ are the mode functions with the lowest and the next lowest eigenfrequencies. Note that $e_{0}(\ell)$ and $e_{1}(\ell)$ are even and odd functions of $\ell$, respectively. Plugging (B8) into the quadratic Lagrangian (B4), we find

$$
\frac{S^{(2)}}{\mathcal{T}}=\int d t \sum_{n=0,1}\left(\dot{c}_{n}^{2}-\omega_{n}^{2} c_{n}^{2}\right) .
$$

The cubic-order terms of the Lagrangian are given by

$$
\begin{aligned}
\frac{\mathcal{L}^{(3)}}{\mathcal{T}} & =C_{0}(\ell) \xi^{3}+C_{1}(\ell) \xi^{2} \xi^{\prime}+C_{2}(\ell) \xi \xi^{2}+C_{3}(\ell) \xi \dot{\xi}^{2} \\
& =\left(C_{0}(\ell)-\frac{1}{3} C_{1}^{\prime}(\ell)\right) \xi^{3}+C_{2}(\ell) \xi \xi^{\prime 2}+C_{3}(\ell) \xi \dot{\xi}^{2}
\end{aligned}
$$

where the second equality holds up to a surface term. Then the cubic order part of the action is given by

$$
\begin{aligned}
\frac{S^{(3)}}{\mathcal{T}} & =\int d t \int_{-\infty}^{\infty} d \ell\left\{\left[\left(C_{0}-\frac{C_{1}^{\prime}}{3}\right) e_{0}^{3}+C_{2} e_{0} e_{0}^{\prime}\right] c_{0}^{3}(t)\right. \\
& +\left[3\left(C_{0}-\frac{C_{1}^{\prime}}{3}\right) e_{0} e_{1}^{2}+C_{2}\left(e_{0} e_{1}^{\prime 2}+2 e_{0}^{\prime} e_{1} e_{1}^{\prime}\right)\right] c_{0}(t) c_{1}^{2}(t) \\
& \left.+C_{3}\left(e_{0}^{3} c_{0} \dot{c}_{0}^{2}+e_{0} e_{1}^{2} c_{0} \dot{c}_{1}^{2}+2 e_{0} e_{1}^{2} \dot{c}_{0} c_{1} \dot{c}_{1}\right)\right\}
\end{aligned}
$$

where we retained only even functions of $\ell$ in the integrand since the odd part does not give a contribution after the integration. We can evaluate the coefficients appearing in this action numerically. For example, when the tip of the string is at $r=r_{0}=1.1$, the action is given by

$$
\begin{aligned}
\frac{S}{\mathcal{T}}= & \int d t \sum_{n=0,1}\left(\dot{c}_{n}^{2}-\omega_{n}^{2} c_{n}^{2}\right)+11.3 c_{0}^{3}+21.5 c_{0} c_{1}^{2} \\
& +10.7 c_{0} \dot{c}_{0}^{2}+3.32 c_{0} \dot{c}_{1}^{2}+6.64 \dot{c}_{0} c_{1} \dot{c}_{1},
\end{aligned}
$$

where $\omega_{0}^{2}=-1.40$ and $\omega_{1}^{2}=7.57$.

The action (B12) describes the motion of the string in a trapping potential with an unstable equilibrium point on its edge, as explained above. One problem of this action is that the kinetic term of the Hamiltonian constructed from this action is not positive definite in some regions within the trapping potential; hence the time evolution there is not well-posed. One way to ameliorate this problem is to apply the following variable change:

$$
c_{0}=\tilde{c}_{0}+\alpha_{1} \tilde{c}_{0}^{2}+\alpha_{2} \tilde{c}_{1}^{2}, \quad c_{1}=\tilde{c}_{1}+\alpha_{3} \tilde{c}_{0} \tilde{c}_{1}
$$

to the action and neglect $\mathcal{O}\left(\tilde{c}_{i}{ }^{4}\right)$ terms, where $\alpha_{i}$ are constants. By taking appropriate values for $\alpha_{i}$, we can ensure that the kinetic term is positive definite within the trapping potential. An example of such a choice is $\alpha_{1}=-1.5, \alpha_{2}=-0.5$ and $\alpha_{3}=-1$, which gives

$$
\begin{aligned}
\frac{S}{\mathcal{T}}= & \int d t \sum_{n=0,1}\left(\dot{\tilde{c}}_{n}^{2}-\omega_{n}^{2} \tilde{c}_{n}^{2}\right)+7.11 \tilde{c}_{0}^{3}+35.3 \tilde{c}_{0} \tilde{c}_{1}^{2} \\
& +4.66 \tilde{c}_{0} \dot{\tilde{c}}_{0}^{2}+1.32 \tilde{c}_{0} \dot{\tilde{c}}_{1}^{2}-7.57 \dot{\tilde{c}}_{0} \tilde{c}_{1} \dot{\tilde{c}}_{1},
\end{aligned}
$$

for which the time evolution within the trapping potential is well-posed. This is the cubic order action (25) studied in Sec. III, and it exhibits chaos for orbits near the unstable maximum corresponding to the black hole horizon.
[1] J. M. Maldacena, Int. J. Theor. Phys. 38, 1113 (1999); Adv. Theor. Math. Phys. 2, 231 (1998).

[2] S. H. Shenker and D. Stanford, J. High Energy Phys. 12 (2014) 046.

[3] S. H. Shenker and D. Stanford, J. High Energy Phys. 03 (2014) 067.

[4] S. Leichenauer, Phys. Rev. D 90, 046009 (2014).
[5] A. Kitaev, Talk Given at Fundamental Physics Symposium, 2014.

[6] S. H. Shenker and D. Stanford, J. High Energy Phys. 05 (2015) 132.

[7] S. Jackson, L. McGough, and H. Verlinde, Nucl. Phys. B901, 382 (2015).

[8] J. Polchinski, arXiv:1505.08108. 
[9] J. Maldacena, S. H. Shenker, and D. Stanford, J. High Energy Phys. 08 (2016) 106.

[10] A. I. Larkin and Y. N. Ovchinnikov, J. Exp. Theor. Phys. 28, 1200 (1969).

[11] P. Basu, D. Das, and A. Ghosh, Phys. Lett. B 699, 388 (2011).

[12] P. Basu and L. A. Pando Zayas, Phys. Lett. B 700, 243 (2011); , Phys. Rev. D 84, 046006 (2011).

[13] P. Basu, D. Das, A. Ghosh, and L. A. Pando Zayas, J. High Energy Phys. 05 (2012) 077; L. A. Pando Zayas and D. Reichmann, J. High Energy Phys. 04 (2013) 083.

[14] A. Stepanchuk and A. A. Tseytlin, J. Phys. A 46, 125401 (2013); Y. Chervonyi and O. Lunin, J. High Energy Phys. 02 (2014) 061.

[15] D. Giataganas, L. A. Pando Zayas, and K. Zoubos, J. High Energy Phys. 01 (2014) 129.

[16] D. Giataganas and K. Sfetsos, J. High Energy Phys. 06 (2014) 018; X. Bai, J. Chen, B. H. Lee, and T. Moon, J. Korean Phys. Soc. 68, 639 (2016).

[17] Y. Asano, D. Kawai, H. Kyono, and K. Yoshida, J. High Energy Phys. 08 (2015) 060.

[18] K. L. Panigrahi and M. Samal, Phys. Lett. B 761, 475 (2016).

[19] L. A. Pando Zayas and C. A. Terrero-Escalante, J. High Energy Phys. 09 (2010) 094; P. Basu, P. Chaturvedi, and P. Samantray, Phys. Rev. D 95, 066014 (2017).

[20] Y. Asano, H. Kyono, and K. Yoshida, J. High Energy Phys. 09 (2016) 103.

[21] T. Ishii, K. Murata, and K. Yoshida, Phys. Rev. D 95, 066019 (2017).

[22] K. Hashimoto and N. Tanahashi, Phys. Rev. D 95, 024007 (2017).

[23] T. Ishii and K. Murata, J. High Energy Phys. 06 (2015) 086.

[24] T. Ishii and K. Murata, J. High Energy Phys. 03 (2016) 035.

[25] J. M. Maldacena, Phys. Rev. Lett. 80, 4859 (1998).

[26] S. J. Rey and J. T. Yee, Eur. Phys. J. C 22, 379 (2001).

[27] S. J. Rey, S. Theisen, and J. T. Yee, Nucl. Phys. B527, 171 (1998).
[28] D. E. Kharzeev, Phys. Rev. D 90, 074007 (2014).

[29] K. Hashimoto and D. E. Kharzeev, Phys. Rev. D 90, 125012 (2014).

[30] I. Iatrakis and D. E. Kharzeev, Phys. Rev. D 93, 086009 (2016).

[31] K. B. Fadafan and S. K. Tabatabaei, Phys. Rev. D 94, 026007 (2016).

[32] L. Bellantuono, P. Colangelo, F. De Fazio, F. Giannuzzi, and S. Nicotri, Phys. Rev. D 96, 034031 (2017).

[33] H. Iida, T. Kunihiro, B. Mueller, A. Ohnishi, A. Schaefer, and T. T. Takahashi, Phys. Rev. D 88, 094006 (2013).

[34] H. Tsukiji, H. Iida, T. Kunihiro, A. Ohnishi, and T. T. Takahashi, Phys. Rev. D 94, 091502 (2016).

[35] H. Tsukiji, T. Kunihiro, A. Ohnishi, and T. T. Takahashi, Prog. Theor. Exp. Phys. (2018) 013D02.

[36] K. Fukushima, Phys. Rev. C 89, 024907 (2014).

[37] K. Fukushima, Rep. Prog. Phys. 80, 022301 (2017).

[38] K. Hashimoto, S. Kinoshita, K. Murata, and T. Oka, Phys. Lett. B 746, 311 (2015).

[39] K. Hashimoto, S. Kinoshita, K. Murata, and T. Oka, Nucl. Phys. B896, 738 (2015).

[40] K. Hashimoto, M. Nishida, and A. Sonoda, J. High Energy Phys. 08 (2015) 135.

[41] K. Hashimoto, K. Murata, and K. Yoshida, Phys. Rev. Lett. 117, 231602 (2016).

[42] R. E. Arias and G. A. Silva, J. High Energy Phys. 01 (2010) 023.

[43] J. L. Albacete, Y. V. Kovchegov, and A. Taliotis, Phys. Rev. D 78, 115007 (2008).

[44] T. Hayata, K. Nawa, and T. Hatsuda, Phys. Rev. D 87, 101901 (2013).

[45] K. B. Fadafan, D. Giataganas, and H. Soltanpanahi, J. High Energy Phys. 11 (2013) 107.

[46] T. Ishii, S. Kinoshita, K. Murata, and N. Tanahashi, J. High Energy Phys. 04 (2014) 099.

[47] C. Schmidhuber, Nucl. Phys. B467, 146 (1996).

[48] R. G. Cai and N. Ohta, Prog. Theor. Phys. 104, 1073 (2000). 